Revue d'histoire de l'enfance « irrégulière »

Le Temps de l'histoire

9 | 2007

Violences et jeunesse

\title{
Les rébellions juvéniles contre les gendarmes dans la France du premier XIXème siècle (1800-1859)
}

\section{Aurélien Lignereux}

\section{(2) OpenEdition \\ 12 Journals}

Édition électronique

URL : http://journals.openedition.org/rhei/2062

DOI : 10.4000/rhei.2062

ISBN : 978-2-7535-1648-9

ISSN : $1777-540 \mathrm{X}$

Éditeur

Presses universitaires de Rennes

Édition imprimée

Date de publication : 15 novembre 2007

Pagination : 59-77

ISSN : 1287-2431

Référence électronique

Aurélien LignereuX, « Les rébellions juvéniles contre les gendarmes dans la France du premier XIXème siècle (1800-1859) ", Revue d'histoire de l'enfance « irrégulière » [En ligne], 9 | 2007, mis en ligne le 01 novembre 2009, consulté le 04 décembre 2020. URL : http://journals.openedition.org/rhei/2062 ; DOI : https://doi.org/10.4000/rhei.2062 


\section{Les rébellions juvéniles contre les gendarmes dans la France du premier XIXème siècle (1800-1859)}

En dépit d'images fortes - celle de la jeunesse des écoles bravant la gendarmerie royale sous le soleil de juillet $1830{ }^{(2)}$ ou encore celle de Guignol, marionnette d'un jeune canut, rossant le Gendarme pour la plus grande joie d'un public enfantin -, ${ }^{(3)}$ la violence juvénile, dans sa dimension collective et contestataire, reste mal connue au siècle de Gavroche. De fait, si l'on dispose de repères concernant les bandes d'enfants ou les révoltes d'adolescents dans les collèges, ${ }^{(4)}$ l'attention s'est surtout portée sur des formes individuelles de délinquance en ce qui concerne leurs jeunes aînés.(5) Pourtant, avec quelque 623 cas reconstitués de 1800 à 1859, les rébellions collectives de jeunes gens - on entendra par là les célibataires de moins de 30 ans - occupent une place notable au sein d'un phénomène rébellionnaire riche de 3.725 rébellions impliquant trois personnes au moins contre des gendarmes, en France (Seine et Corse exclus). ${ }^{(6)}$

Une affaire sur six est donc le fait d'un groupe de jeunes gens. Peut-on dégager pour autant, dans la nature des heurts, une spécificité de la rébellion juvénile ? Présente-t-elle seulement une unité au-delà de l'âge des rebelles ? Cette identité n'est-elle pas à chercher du côté des instances répressives, dans les représentations des responsables et jusqu'aux pratiques des gendarmes, à la fois victimes et verbalisateurs ? Le terme même de rébellion, par lequel le Code pénal qualifie ces violences, ${ }^{(7)}$ ne doit pas masquer que ces rebelles sont d'abord fidèles à leur communauté d'origine, comme le souligne Jean-Claude Farcy dans son portrait de groupe de la jeunesse rurale :

«Dans le monde rural, les jeunes, loin d'être des rebelles, sont au service d'une communauté villageoise souhaitant préserver son autonomie face aux empiétements croissants de l'État et à la diffusion des normes nationales. À la pointe des révoltes exprimant cette résistance pendant le pre-
Aurélien

Lignereux $^{(1)}$
(1) Ancien élève de

l'École normale supérieure de la rue d'Ulm et agrégé d'histoire. ATER à

l'université de Savoie.

Thèse de doctorat soutenue à l'université du Maine (LHAMANS-

CERHIO), sous la direction de Nadine Vivier et de Jean-Noël Luc, portant sur les résistances opposées à l'essor de l'implantation et des missions de la gendarmerie : Rébellions à la gendarmerie et autorité de l'État dans la France du premier XIXème siècle (1800-1859).

(2) Jean-Claude Caron nuance les mythes contemporains comme les dénégations de l'historiographie anglo-saxonne :

Aurélien Lignereux / p. 59 à 77 
Générations romantiques.

Les étudiants de Paris et

le Quartier Latin (I8I4-

I85I), Paris, Armand

Colin, 1991, 435 p.

(3) Aurélien Lignereux,

« Rosser le gendarme dans

les spectacles de marion-

nettes au XIXème siècle :

une école de rébellion ? ",

in Jean-Noël Luc, [dir.],

"Figures de gendarmes",

Sociétés \& représentations,

$\mathrm{n}^{\circ} 16$, sept. 2003,

p. 97-113.

(4) Frédéric Chauvaud,

«Gavroche et ses pairs :

aspects de la violence

politique du groupe

enfantin en France au

XIXème siècle ", Cultures

et conflits, 1995, $\mathrm{n}^{\circ} 18$,

p. 21-33; Agnès Thiercé,

«Révoltes de lycéens,

révoltes d'adolescents

au XIXème siècle ",

Histoire de l'éducation,

janvier 2001, n 89 ,

p. 53-93.
(5) Frédéric Chauvaud,

«Violence juvénile,

violence familiale

(1830-1880) ", I848.

Révolutions et mutations

au XIXème siècle, 1992 ,

$\mathrm{n}^{\circ} 8$, "Jeunesse au

XIXème siècle ", p. 39-48.

Les monographies

consacrées à l'activité d'un

tribunal ne manquent

pas de répartir la délin-

quance jugée en fonction

des classes d'âge, dans

la continuité du Compte

général de l'administration

de la justice criminelle

en France (moins de

16 ans, 16-20, 21-25,

26-30).

(6) La constitution de

ce corpus, reposant sur

le dépouillement de plus

de 1.300 cartons aux

Archives nationales et au

Service historique de la

défense - Département de

l'armée de terre, est

précisée dans l'introduc-

tion de notre doctorat. mier XIXème siècle, surtout dans les régions montagneuses et les plus éloignées du pouvoir, ils participent à la conservation des coutumes qui leur donnent d'ailleurs un rôle important [...] Tout se passe comme si la violence juvénile, susceptible de naître des rapports sociaux très oppressifs, laissant peu de place à l'initiative individuelle, était canalisée au service de la communauté, contre l'extérieur, le village voisin, les gardes forestiers ou les gendarmes. " ${ }^{(8)}$

À la gendarmerie, bras armé de l'État, s'opposerait donc la jeunesse, fer de lance des sociétés villageoises. Ce serait ainsi la transcription d'un contentieux plus global en ce premier XIXème siècle, qui consacre à la fois l'affirmation de l'État-Nation et la vitalité de sociétés rurales à leur apogée démographique. ${ }^{(9)}$ Affrontement logique mais réducteur, car d'autres facteurs n'interviennent-ils pas dans le cas des groupes de jeunesse, tels que la culture de la force et le goût du défi, l'entraînement collectif et les purs débordements festifs ? Deux approches complémentaires sont alors à mettre en œuvre : l'une panoramique, afin de dégager les caractéristiques d'ensemble des rébellions juvéniles ; l'autre, resserré sur un cadre monographique, pour mieux comprendre les logiques et les rituels des affrontements.

\section{La rébellion juvénile en questions : délimitations du phénomène}

L'éternelle jeunesse ? Les rébellions juvéniles

à l'épreuve de la chronologie

Le premier XIXème siècle français n'a pas l'exclusivité des rixes intercommunales ni des désordres du Carnaval qui virent à l'affrontement avec les forces de l'ordre. Entre la jeunesse du Languedoc qui « mesure sa force " au besoin contre les cavaliers de la maréchaus- 
sée et les jeunes Parisiens qui "se frottent aux sergots » de la Belle Époque, ${ }^{(10)}$ l'uniforme change davantage que la forme des rébellions. L'éternelle jeunesse de ces débordements fait douter de la validité de la lecture historienne, comme Jean Nicolas s'en est précédemment inquiété :

«Saisir la turbulence des jeunes générations sous le seul angle de la violence collective peut paraitre réducteur. [...] sur ce qui provoque le bouillonnement juvénile comme sur ce qu’il révèle, les réponses mettent en jeu la société globale avec ses traditions, ses pratiques intrafamiliales, ses comportements de groupes, bref une immense matière ethnographique et sociologique [...] la rébellion des jeunes constitue d'abord une donnée d'ordre vital, et la contestation de l'autorité qu'elle développe renvoie à des exigences libertaires permanentes, intemporelles. » ${ }^{(11)}$

Néanmoins, une comparaison entre les 634 affaires relevant de la contestation de jeunesse recensées de 1661 à 1789 , et les 623 cas des années 1800 à 1859 , légitime l'approche chronologique. On remarque tout d'abord que les rébellions de la jeunesse pèsent davantage sur le premier XIXème siècle, avec 16,6 \% du total rébellionnaire, alors que, sous l'Ancien Régime, cette proportion descend à 7,4\% des 8528 mouvements populaires identifiés. On serait donc tenté de conclure au rajeunissement de la rébellion, voire de l'interpréter comme l'assagissement du reste des Français : les rébellions collectives deviennent davantage le fait de la seule jeunesse. Ce schéma linéaire est à nuancer. Il ressort en effet que les rébellions juvéniles se manifestent à partir des années 1740, pour être massives dans les années 1770, si bien que $83 \%$ d'entre elles se situent entre 1740 et 1789 . Les rébellions juvéniles du premier XIXème siècle s'inscrivent donc dans une continuité séculaire, puisque plus de $80 \%$ d'entre elles ont lieu avant 1840. Par-delà la fracture révolutionnaire - chaînon manquant faute d'étude spécifique à l'échelle de la France -, se dégage donc un temps fort de la rébellion juvénile des années 1740 aux années 1840 , période qui, entre autres permanences, présente une incontestable cohérence sur le plan démographique, fondée sur la croissance de la population et l'importance consécutive des jeunes classes d'âge.

Cette continuité se retrouve dans les temps et dans les thèmes des rébellions juvéniles : après une première phase tumultueuse à la fin de l'hiver, au temps du Carnaval, les mois d'été sont surreprésentés. Le dimanche domine
(7) Aux termes de l'article $209 \mathrm{du}$ Code pénal de 1810, il y a rébellion en cas de violences et voies de fait exercées à l'encontre d'un agent de l'autorité publique dans l'exercice de ses fonctions.

(8) Jean-Claude Farcy, La jeunesse rurale dans la France du XIXème siècle, Paris, Éd. Christian, 2004, p. 145 et 181.

(9) L'historiographie s'accorde pour dater de la fin des années 1850 l'effacement des révoltes rurales : Alain Corbin, "Histoire de la violence dans les campagnes françaises au XIXème siècle. Esquisse d'un bilan ", Éthnologie française, 1991, t. XXI, n 3, p. 224-236, et « La violence rurale dans la France du XIXème siècle et son dépérissement : l'évolution de l'interprétation politique ", in Philippe Braud, [dir.], La violence politique dans 
les démocraties européennes occidentales, Paris,

L'Harmattan, 1993,

p. 61-73; Frédéric

Chauvaud, "Le dépérissement des émotions paysannes dans les territoires boisés au XIXème siècle ", in Alain Plessis et de JeanClaude Farcy, [dir.], La terre et la cité. Mélanges offerts à Philippe Vigier, Paris, Créaphis, 1994, p. 101-114.

(10) Nicole Castan, Les criminels de Languedoc: les exigences d'ordre et les voies du ressentiment dans une société prérévolutionnaire (I750-I790), Publications de l'université de Toulouse-Le Mirail, 1980, p. 195 ; Francis Demier, « Délinquants à Paris, à la fin du XIXème siècle ", Recherches contemporaines, 1997, n 4, p. 234.

(11) Jean Nicolas, $\mathrm{La}$ rébellion française. Mouvements populaires et conscience sociale (I66I- nettement : $37 \%$ des rébellions juvéniles d'Ancien Régime ; $36 \%$ de celles du premier XIXème siècle (29\% des autres). D'un siècle à l'autre, la défense de valeurs conservatoires l'emporte sur les revendications contestataires. Les différences méthodologiques rendent toutefois hasardeux un rapprochement méthodique. D’une part, Jean Nicolas englobe tout le champ de la protestation collective pourvu que quatre émeutiers au moins y participent, alors que nous nous bornons aux affrontements opposant des gendarmes à trois rebelles et plus. D'autre part, Jean Nicolas suit une logique thématique qui associe à la jeunesse une série de conflits certes typés (charivaris, rixes entre paroisses, heurts avec la maréchaussée, tumultes écoliers, agitation nocturne, etc.), mais qui, dans les faits, ne sont pas tous l'apanage de la jeunesse. Au contraire, sont ici considérées comme juvéniles les rébellions dont la plupart des acteurs sont des jeunes gens. Si l'on y perd en cohésion, ce choix a cependant un double mérite : il n'exclut pas a priori des rébellions prises en charge par la jeunesse, en dehors de son champ d'action traditionnel; il inclut des affaires connotées juvéniles, mais pour lesquelles il s'avère que la participation des jeunes gens a été minoritaire.

La comparaison systématique doit se limiter au premier XIXème siècle, entre les 623 rébellions juvéniles et les 3.102 autres. Le rythme des affrontements offre une première donnée $[c f . f i g . I]$.

Jusqu'en 1828, un parallélisme s'observe entre les deux courbes. Puis les rébellions juvéniles se tassent, alors que s'attisent au contraire les contrastes de l'ensemble rébellionnaire. Les rébellions juvéniles sont insensibles au temps court : les flambées de 1815-1817, 1829-1836, 1846-1849 sont littéralement gommées. Les troubles économiques, sociaux et politiques débordent de loin les colères juvéniles. Ces dernières éclatent en réponse à des pressions spécifiques : au début du Premier Empire, lorsque les brigades réorganisées sont en mesure de s'attaquer aux groupes de conscrits; à la fin du règne de Napoléon, lorsque ceux-ci rejettent ouvertement les exigences démesurées d'un pouvoir chancelant ; à partir de 1818 et jusqu'au milieu des années 1820, lorsque la loi Gouvion-Saint-Cyr se heurte au refus d'une partie de la jeunesse convoquée aux tirages au sort, puis regroupée en bandes d'insoumis, cette agitation juvénile est alors redoublée par les rixes intercommunales, qui connais- 
Fig 1. - Rythme annuel des rébellions juvéniles au sein des rébellions collectives, I800-1859

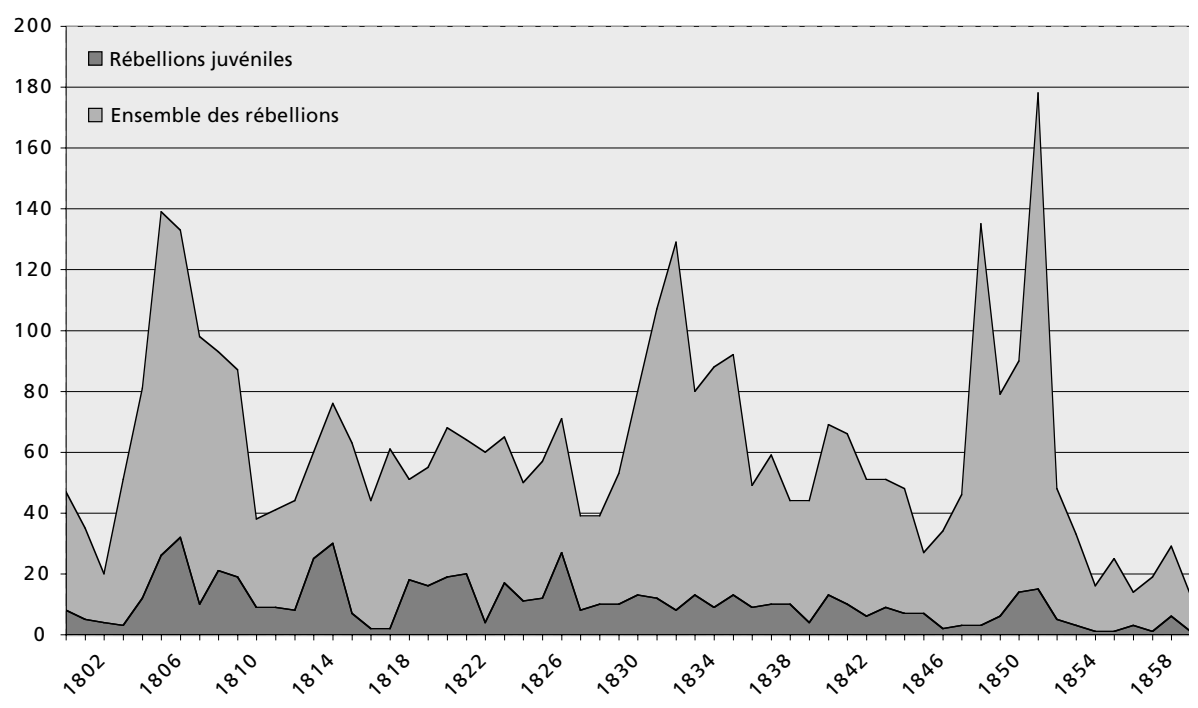

sent leur apogée. ${ }^{(12)}$ Quant au regain des années 1850-1851 (décembre mis à part), avant de l'attribuer à la politisation de la jeunesse, il semble plus juste de le mettre en rapport avec la compression mise en œuvre sous la République conservatrice. Inquiets des progrès des " rouges », les autorités recommandent aux gendarmes une surveillance appuyée des rassemblements, les fêtes en particulier, multipliant de ce fait les occasions de conflit avec une jeunesse gênée dans ses plaisirs traditionnels.

\section{Un répertoire spécifique?}

Les rébellions juvéniles à l'aune du phénomène rébellionnaire

Le profil de la rébellion juvénile type conforte ces enseignements. Chacune des 3.725 affaires a fait l'objet d'une fiche exploitant les données disponibles à partir d'un questionnement ramifié en 57 rubriques. Des écarts significatifs dessinent les contours propres aux 623 rébellions juvéniles. On retiendra ainsi leur localisation privilégiée dans les communes rurales (moins de 2.000 habitants),
I789), Paris, Seuil, 2002,

p. 441. Le développement suivant s'appuie sur le chapitre « Jeunesse, jeunesse... ", p. 441-482.

(12) François Ploux, Guerres paysannes en Quercy. Violences, conciliations et répression pénale dans les campagnes $d u$ Lot (I810-I860), Paris, La boutique de l'histoire, 2002, 376 p. 
cadre de deux affaires sur trois, contre trois sur cinq pour les autres rébellions. Il est d'ailleurs à souligner que le quart des affrontements entre des jeunes et des gendarmes ont lieu en marge du village, dans un bois ou sur une route, ce qui renvoie à la marginalité des bandes poursuivies par les gendarmes. De fait, les rébellions juvéniles sont surreprésentées dans les espaces montagnards et dans le bocage de l'Ouest, terres de prédilection des bandes d'insoumis.

Fig. 2. - Répartition départementale des rébellions juvéniles au sein des rébellions collectives, I800-1859

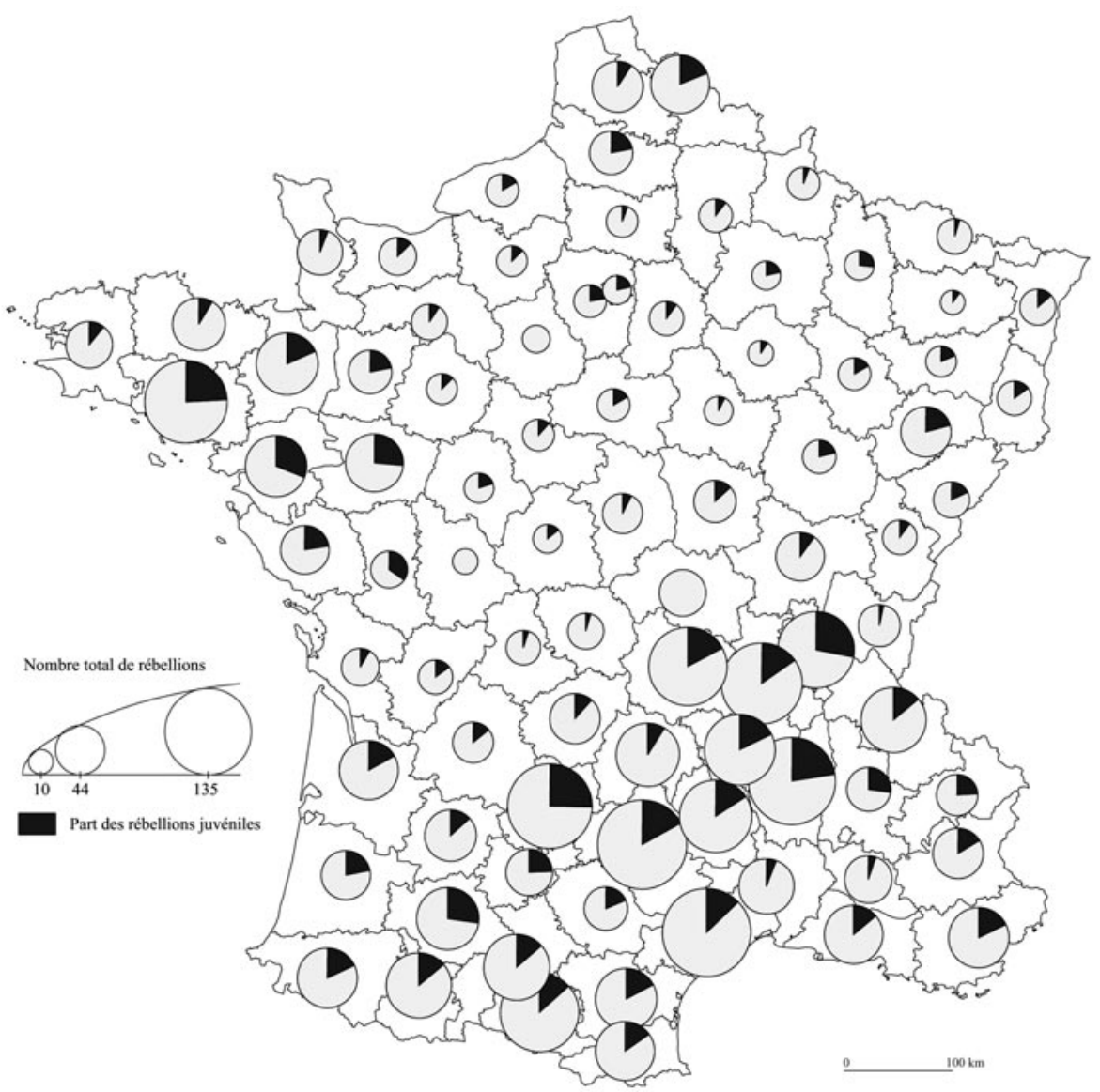


En bref, cette carte offre une nouvelle illustration de la ligne Saint-Malo/ Genève, à ceci près que le Centre-Ouest se rattache à la France du Nord-Est en raison du calme de leurs populations. La répartition des rébellions juvéniles attise cette frontière intérieure, au point que plus un département compte d'affrontements et plus la proportion des luttes de la jeunesse est élevée : par exemple, l'Ardèche, le Lot ou le Morbihan se détachent à la fois en raison du grand nombre des affaires et du taux atteint par les rébellions juvéniles.

Cette accentuation du modèle rébellionnaire est manifeste en ce qui concerne le contexte. Avec 29 \% des affaires, la fête est en effet le temps privilégié de l'affirmation collective et violente de la jeunesse. Si l'on y ajoute les soirs de bal, les jours de foire et de tirage au sort, on atteint $58 \%$, alors que seules $26 \%$ des 3.102 autres rébellions ont lieu lors de telles occasions. D'où ce constat : si la conjoncture globale n'incite nullement aux rébellions juvéniles, celles-ci doivent beaucoup aux circonstances locales favorables aux rassemblements. L'alcool joue donc son rôle : dans $42 \%$ des cas, les jeunes gens sont pris de boisson, pourcentage qui descend à $16 \%$ pour les autres rébellions.

Fig. 3-Cause des 623 rébellions juvéniles
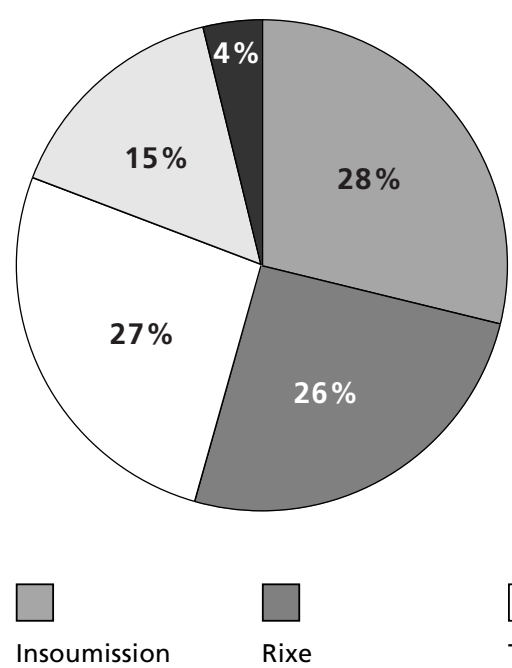

Fig. 4 - Causes des 3102

autres rébellions
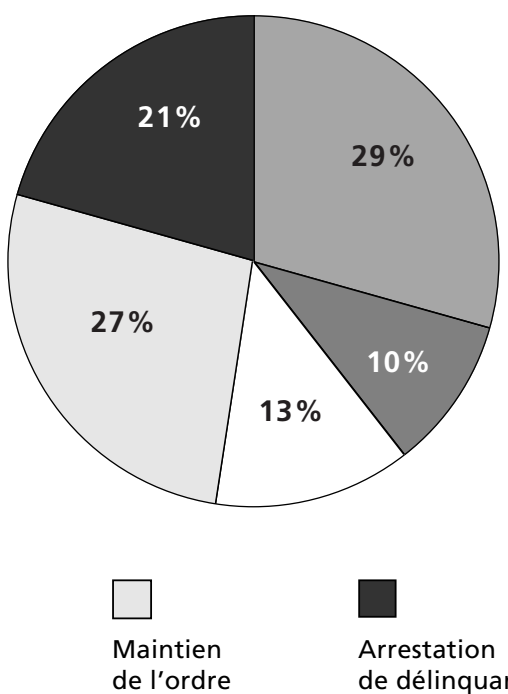

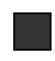

Arrestation de délinquants 
(13) Mansuétude que relève par exemple Vincent Robert, "Aux origines de la manifestation en France (17891848) ", in Pierre Favre, [dir.], La manifestation, Paris, Presses de la FNSP, 1990, p. 76-77.
Trois sujets de griefs polarisent les rébellions juvéniles : le rejet du service militaire, les rixes et les débordements turbulents (charivari, tapage ou fermeture de cabaret). Ces deux derniers ensembles assurent la spécificité des rébellions de jeunesse, avec $52 \%$ des causes contre $23 \%$ pour les autres rébellions.

Encore ces graphiques ne mentionnent-ils que les principales familles de conflit. Leur ramification fait ressortir une nette simplification des causes. Douze sujets de friction relatifs à des opérations de maintien de l'ordre (frumentaire, antifiscale, etc.) et à des arrestations de délinquants (chasseurs, contrebandiers, etc.) n'occasionnent au total que 2,5\% des rébellions de jeunes, alors que cette part est multipliée par dix dans le cas des 3102 autres affaires. Quant à la dimension politique qui peut animer les rébellions, il est à noter que, contrairement au trompe-l'œil romantique - et, en tout cas, hors de l'exception parisienne -, les luttes de la jeunesse ont plus rarement une couleur partisane affichée (14\% contre $18 \%$ ) et, le cas échéant, elle est massivement légitimiste avec près des deux tiers des rébellions juvéniles politisées. Cette orientation ne surprend guère dans la mesure où le basculement de jeunes gens dans la rébellion naît surtout du rejet de la conscription napoléonienne ou de la Monarchie de Juillet qui, dans ses premières années, a érigé le recrutement en test politique.

Cette inflexion propre au déclenchement des rébellions juvéniles se prolonge dans le déroulement et dans le dénouement des heurts [cf. fig. s].

Ce tableau révèle une violence redoublée dans le cas des rébellions juvéniles. Ce constat est d'autant plus net que les deux types de rebelles disposent d'un armement équivalent. C'est leur usage effectif qui differe, comme l'illustre celui des armes à feu : les jeunes font feu plus de quatre fois sur cinq, alors que leurs aînés ne tirent que dans 55 \% des cas, ce qui dénote ou bien une perte de sang-froid ou bien la radicalité résolue des groupes de jeunes gens, dont une partie - insoumis ou chouans - sont en rupture de ban. Cette violence, née de l'exaspération face aux poursuites dont ils sont l'objet, nourrit celle des gendarmes. La riposte frappe en effet par sa sévérité ; on notera par exemple le large recours qui est fait du sabre. D'où un tribut plus lourd au sein des groupes de jeunesse : avec 71 tués, ils déplorent $28 \%$ du total des pertes des rebelles, pour $16,5 \%$ seulement des rébellions. Ce bilan prouve que, contrairement à la retenue dont ils peuvent témoigner face à des étudiants, ${ }^{(13)}$ 
Fig. 5. Rapports de force et issues comparées des rébellions

\begin{tabular}{|c|c|c|}
\hline & $\begin{array}{l}\text { Ensemble des } 3.102 \text { affaires } \\
\text { hors rébellions juvéniles }\end{array}$ & $\begin{array}{l}\text { Ensemble des } 623 \\
\text { rébellions juvéniles }\end{array}$ \\
\hline $\begin{array}{l}\text { Taille estimée de } \\
\text { l'attroupement }\end{array}$ & $\begin{array}{r}3-10: 25 \% \\
11-50: 23 \% \\
51-200: 37 \% \\
>200: 15 \%\end{array}$ & $\begin{array}{r}3-10: 38 \% \\
11-50: 32 \% \\
51-200: 26 \% \\
>200: 4 \%\end{array}$ \\
\hline $\begin{array}{r}\text { Armes } \\
\text { des rebelles }\end{array}$ & $\begin{array}{r}\text { Force physique seule : } 34 \% \\
\text { Pierres : } 26 \% \\
\text { Outils, bâtons : } 20 \% \\
\text { Armes à feu : } 16 \% \\
\text { (tirs effectifs : } 55 \% \text { ) } \\
\text { Armes blanches : } 4 \%\end{array}$ & $\begin{array}{r}\text { Force physique seule : } 35 \% \\
\text { Pierres : } 24 \% \\
\text { Outils, bâtons : } 20 \% \\
\text { Armes à feu : } 17 \% \\
\text { (tirs effectifs : } 81 \% \text { ) } \\
\text { Armes blanches : } 4 \%\end{array}$ \\
\hline $\begin{array}{r}\text { Armes } \\
\text { des gendarmes }\end{array}$ & $\begin{array}{c}\text { Force physique seule : } 40,5 \% \\
\text { Absence de riposte : } 30 \% \\
\text { Armes blanches : } 19 \% \\
\text { (employées : } 1 \text { fois sur } 3 \text { ) } \\
\text { Armes à feu : } 10,5 \% \\
\text { (tirs effectifs : } 7 \text { fois sur } 10 \text { ) }\end{array}$ & $\begin{array}{l}\text { Force physique seule : } 41 \% \\
\text { Absence de riposte : } 21 \% \\
\text { Armes blanches : } 23 \% \\
\text { (employées : } 2 \text { fois sur 3) } \\
\text { Armes à feu : } 15 \% \\
\text { (tirs effectifs : } 4 \text { fois sur } 5 \text { ) }\end{array}$ \\
\hline $\begin{array}{r}\text { Pertes } \\
\text { des rebelles }\end{array}$ & $\begin{array}{l}: 180 \text { tués } \\
\text { (5,8\% affaires) }\end{array}$ & $\begin{array}{l}: 71 \text { jeunes tués } \\
\text { (11,4\% affaires) }\end{array}$ \\
\hline $\begin{array}{r}\text { Pertes } \\
\text { des gendarmes }\end{array}$ & $\begin{array}{l}: 75 \text { tués } \\
\text { (2,4\% affaires) }\end{array}$ & $\begin{array}{l}\text { : } 31 \text { tués } \\
\text { (4,9\% affaires) }\end{array}$ \\
\hline $\begin{array}{r}\text { Issue } \\
\text { des rébellions }\end{array}$ & $\begin{array}{r}\text { Défaite des gendarmes : } 58 \% \\
\text { Défaite des rebelles : } 42 \%\end{array}$ & $\begin{array}{l}\text { Défaite des gendarmes : } 41 \% \\
\text { Défaite des rebelles : } 59 \%\end{array}$ \\
\hline
\end{tabular}


(14) À titre d'illustration, à Aix, le 4 juin 1820, des étudiants en droit tiennent en échec trois quarts d'heure durant les gendarmes qui veulent conduire un des leurs chez le juge d'instruction. Ses camarades bloquent la prison en formant à trois reprises un barrage humain, sans jamais frapper ni insulter les gendarmes, de sorte que leur résistance ne peut pas être qualifiée de rébellion; le procureur général d'Aix au ministre de la Justice, 5 et 18 juillet 1820, AN, $\mathrm{BB}^{18} 1064$

(15) Procès-verbal de la brigade de Clermont, 28 janvier 1813, AN, F7 8.

(16) Le procureur général de Rennes au ministre de la Justice, 5 juillet 1837, AN, les gendarmes hésitent moins à porter des coups contre de jeunes paysans, qui forment l'ossature de notre corpus. Encore faut-il préciser que cette différenciation sociale reflète peut-être d'abord le type d'atteintes dont ils sont l'objet. ${ }^{(14)}$

En tout cas, cette détermination des gendarmes s'avère efficace : alors qu'ils s'inclinent dans $58 \%$ des autres confrontations, ils sont défaits dans seulement $41 \%$ des rébellions juvéniles. La vigueur physique de la jeunesse se retourne contre elle dans la mesure où les gendarmes ne reculent pas devant l'escalade. La violence des jeunes gens ne suffit pas à compenser leurs effectifs bien inférieurs à ceux des autres groupes rebelles : ces derniers dépassent la cinquantaine d'attroupés plus d'une fois sur deux, ce qui n'est le cas que dans $30 \%$ des rébellions juvéniles. Du reste, la force d'un rassemblement ne tient pas au seul poids du nombre, mais elle repose aussi sur l'effet moral qui résulte de l'implication de toute une communauté. La part importante des rébellions spécifiques aux jeunes gens ne trahit-elle pas leur isolement ? Ou bien est-ce au contraire le résultat d'une répartition des rôles, comme à la suite de l'arrestation d'un déserteur à Blanzat, près de Clermont, en janvier 1813, où, au dire des gendarmes, "les femmes invitaient la jeunesse à empêcher que nous l'emmenions " ? (15)

\section{" Il faut que jeunesse se passe »...}

\section{La disqualification des rébellions juvéniles à l'épreuve des faits}

La thèse de l'isolement est privilégiée par les autorités. De fait, plus que tout autre type de rébellion, les désordres juvéniles sont interprétés comme relevant en quelque sorte de l'ordre des choses. Par-delà les changements de régime, un même souci prévaut : affirmer le caractère naturel de ces troubles afin de les neutraliser. La dominante juvénile d'une rébellion est relevée pour en limiter la gravité. L'esprit d'insubordination prêté à la jeunesse n'inquiète pas outre mesure, car il est dissocié de l'entreprise de subversion. En juillet 1837, un déserteur est arraché aux gendarmes lors d'une embuscade près d'Auray. Ayant redouté une résurgence de la chouannerie, le procureur général respire à l'annonce que les suspects sont des amis du déserteur : "Ce coup de main deviendrait alors plutôt une démonstration d'affection locale, une camaraderie de village, qu'un nouveau symptôme politique. " ${ }^{(16)}$ Après une émeute, le 15 mars 1851, à Saint-Amanden-Puisaye dans la Nièvre, on souligne que la rébellion a eu lieu la veille du tirage 
au sort et qu'en définitive " cette rébellion a sa cause plus dans des circonstances fortuites que dans des combinaisons de parti. Le parti pris de quelques mauvais sujets, l'humeur tapageuse d'une nombreuse jeunesse, la contagion de l'exemple, suffisent à tout expliquer $»{ }^{(17)}$

La caractérisation de la rébellion juvénile s'effectue sur le mode de la disqualification, modulée au gré des circonstances : jeunesses "belliqueuses », lors de rixes intercommunales; jeunesse "indisciplinée » ou " tumultueuse ", en cas de charivaris ou d'autres tapages $;^{(18)}$ " mauvais garnements » ou « mauvais drôles ", lorsque les faits confinent à la délinquance ; «étourdis » ou « brouillons », pour discréditer les manifestations politiques de la jeunesse au théâtre ou sur les boulevards des villes... Du reste, ce genre d'association ne leur est pas spécifique : les classes « laborieuses " ne sont-elles pas « dangereuses »?

De même, le discours dominant aime à opposer la turbulence juvénile à l'expérience de la gendarmerie, surtout à partir des années 1840 quand se généralise une nouvelle image du gendarme, présenté comme un vieux soldat aguerri en même temps qu'un bon père de famille, dévoué et désintéressé. Le compte rendu que fait la Gazette des tribunaux du 17 janvier 1847 du jugement correctionnel de douze rebelles de Fontenay-aux-Roses (âgés de 17 à 21 ans), est emblématique : "Depuis quelques mois déjà, une sourde rumeur grondait parmi cette turbulente jeunesse à l'endroit des gendarmes de Châtillon [...] Ces derniers, plus sages que leurs adversaires, apportaient dans ce triste conflit toute la prudence, toute la longanimité possible. »

De fait, ce sont des hommes déjà mûrs, mûris en tout cas par une carrière préalable à l'armée, qui intègrent la gendarmerie, à 29 ans en moyenne. ${ }^{(19)}$ Cet indicateur reste ambivalent : faut-il voir dans la différence d'âge un facteur d'incompréhension supplémentaire, ou bien l'expérience des gendarmes ne joue-t-elle pas dans le sens de la prévention, comme le prouvent a contrario les incidents suscités par de jeunes chefs de brigade, maladroits dans leur quête d'autorité ?

Quoi qu'il en soit, la jeunesse des rebelles fournit à des autorités volontiers paternalistes un moyen de pression sur les communautés villageoises. Des préfets peuvent mettre en demeure une commune de réprimer elle-même les excès de ses jeunes. La période napoléonienne, dont les méthodes répressives s'appuient sur la responsabilité collective, se distingue par ces rappels à l'ordre. En 1804, il y aurait ainsi, selon le préfet Chazal, cinq ou six mauvaises communes
$\mathrm{BB}^{18} 1247$.

(17) Le procureur général de Bourges au ministre de la Justice, 20 mars $1851, \mathrm{AN} \mathrm{BB}^{30} 392$.

(18) On retrouve souvent terme à terme les réactions ambivalentes d'Ancien Régime. Frédérique Pitou, " Jeunesse et désordre social : les coureurs de nuit à Laval au XVIIIème siècle ", RHMC, t. 47-1, janvier-mars 2000, "Ordres et désordres, XVIIème-XXème siècles ", p. 69-92.

(19) Pour les premiers éléments du portrait social du gendarme, Jean-Noël Luc, « Le gendarme, "un soldat qui a pris racine" ? ", in Jean-Noël Luc, [dir.], Gendarmerie, État et société au XIXème siècle, Paris, Publications de la 
Sorbonne, 2002, p. 320.

(20) Le préfet des

Hautes-Pyrénées au conseiller d'État chargé du 2ème arrondissement de police, 13 fructidor an XII (31 août 1804), AN, $\mathrm{F}^{7} 8479$

(21) Le préfet des Bouches-du-Rhône au conseiller d'État chargé du 2ème arrondissement de police, 7 juin 1806, AN, $\mathrm{F}^{7} 8416$.

(22) Les résultats reposent sur 683 affaires jugées de 1800 à 1859 , tant en cour d'assises qu'en tribunal correctionnel, mais aussi dans les cours de justice criminelle spéciale ou dans les cours prévôtales. Cela constitue un sondage de l'ordre d'un cinquième des 3725 rébellions. La présentation de ces jugements est détaillée dans notre thèse. dans les Hautes-Pyrénées : «Une jeunesse indisciplinée les domine, et presque personne n'ose se plaindre de ses excès. " D'où une série de tapages qui aboutissent à une rébellion contre la gendarmerie à Juillan, dans la soirée du 28 août. ${ }^{(20)}$ À Barbentane, après l'enlèvement d'un déserteur en mai 1806 par un groupe armé, Thibaudeau, préfet des Bouches-du-Rhône, fait désarmer la commune sous la surveillance d'une centaine de soldats : "Cette leçon leur a fait sentir à tous qu'il ne suffisait pas de rester paisibles spectateurs des scènes d'insurrection de la part d'une jeunesse indocile et réfractaire aux lois, mais qu'il fallait encore les persuader à l'obéissance et s'opposer aux actes d'insubordination. " ${ }^{(21)}$ Ces efforts pour dissocier les jeunes de leur communauté sont voués à échouer face aux réalités : à Barbentane, les coupables sont certes des conscrits réfractaires, mais des classes de l'an 7 et de l'an 8, devenus entre-temps des hommes mariés et pères de famille.

La teneur des jugements fournit un bon repère pour apprécier les réactions que suscitent les rébellions juvéniles. L'étude d'un échantillon d'affaires apporte sur ce point des enseignements partiels mais suggestifs. ${ }^{(22)}$ Il convient tout d'abord de s'intéresser à l'instruction. Les procédures aboutissent en effet à une distorsion significative : bien que les rébellions juvéniles ne pèsent que pour un sixième des affaires, les jeunes représentent le quart des prévenus. Cette surreprésentation est d'autant plus remarquable que les attroupements juvéniles sont de plus faible envergure [cf. fig. s]. Il est donc permis de conclure ou bien à des poursuites plus systématiques à l'encontre des bandes de jeunes gens en raison de leur dangerosité supposée, ou bien à une moindre habileté de ces derniers à mettre en défaut l'instruction, ce qui est peut-être lié à un moindre investissement de la communauté villageoise en leur faveur. Les jeunes prévenus préferent assurer leur salut par la fuite, comme l'indique un plus fort taux de contumax dans leurs rangs.

Les peines prononcées confirment la spécificité du traitement réservé aux jeunes gens. Ils ne sont que $29 \%$ à bénéficier d'un acquittement, alors que $43 \%$ des prévenus des autres rébellions sont absous. Toutefois, cette fermeté ne se confond pas avec la sévérité. En effet, seuls $16 \%$ des jeunes condamnés le sont à une peine d'emprisonnement supérieure à deux ans, ce qui est le cas de $23 \%$ des condamnés pour les autres rébellions. En matière de rébellions juvéniles, la fréquence et la faiblesse relative des peines (64\% ne dépas- 
sent pas six mois de prison, $20 \%$ sont comprises entre six mois et deux ans) autorisent par conséquent à parler d'une pratique pédagogique du châtiment. Celui-ci s'apparente souvent à un avertissement, en partie grâce à un usage extensif de l'article 463 du Code pénal sur les circonstances atténuantes.

La singularité statistique des rébellions juvéniles au sein du répertoire rébellionnaire global est ainsi acquise. L'échelle du récit doit permettre désormais de mieux en comprendre les ressorts.

\section{Jeunesses rebelles en action :}

\section{l'exemple du département du Rhône sous la Restauration \\ État des lieux et éventail des situations}

En effet, un cadre monographique est plus adéquat pour restituer une plus juste mesure des scénarios types. Reste à en délimiter les cadres. Sous l'Empire, la pression sur la jeunesse est intense. Les pamphlets de la légende noire auront beau jeu de dénoncer le rapt des garçons, propre à donner une nouvelle jeunesse au mythe de l'ogre. Combien de fois les gendarmes interpellent un individu sur le seul critère qu'il leur paraît " de l'âge de la conscription "! Autant dire qu'il y a comme un délit de jeunesse, risque d'arrestation tempéré il est vrai par la solidarité locale. La communauté, et d'abord la famille, a besoin de la force de travail de ses jeunes. Ce ne sont cependant pas ces rébellions accomplies pour la jeunesse qui nous intéressent ici, mais celles menées par la seule jeunesse. La Restauration offre un meilleur poste d'observation. Elle marque un moment charnière de la résistance au recrutement, dont le caractère communautaire s'efface peu à peu; c'est aussi une période de reprise en main religieuse et disciplinaire susceptible de heurter de front la jeunesse. Aussi constate-on que $28 \%$ des rébellions juvéniles se concentrent de 1818 à 1829, années qui ne représentent que $20 \%$ de la période étudiée.

Avec 19 rébellions collectives de jeunes se succédant de 1816 à 1830, le Rhône, en pointe sur la dorsale rebelle qui court du cœur des Pyrénées à Lyon [cf. fig. 2], offre de nombreuses illustrations du phénomène. De plus, ces affaires concernent l'ensemble du département, offrant ainsi une large gamme de situations dans un espace à la géographie contrastée, qui fait qu’à cinq lieues de la seconde ville de France vivent des sociétés villageoises sur un bastion de hautes terres. ${ }^{(23)}$ Les rébellions juvéniles se répartissent dans les communes sub-
(23) Gilbert Garrier,

Paysans du Beaujolais et du Lyonnais (I800-I870), Grenoble, PUG, 
1973, 2 vol.

(24) Tribunal correctionnel de Villefranchesur-Saône : dossier de procédure (1826) ; tribunal correctionnel de Lyon : registre des jugements (1830), A.D. Rhône, Uv 1199 et Ucor 168.

(25) Frédéric Chauvaud en a tenté la cartographie, "Les rixes intervillageoises sous la Restauration. L'impossible dénombrement d'un conflit singulier ", in Benoît Garnot, [dir.], L'infrajudiciaire du Moyen Âge à l'époque contemporaine, Actes du colloque de Dijon, 56 octobre 1995, Dijon, EUD, 1992, p. 437-445.

(26) Procès-verbal de la brigade de Beaujeu, 24 juin 1830, A.D. Rhône, Uv 1206. urbaines de La Guillotière, d'Oullins et de Saint-Clair, dans le Plateau lyonnais (Chaponost), dans les Monts du Lyonnais (Cublize, Larajasse ou Yzeron), dans les Monts du Beaujolais (Chamelet, Marchamp, Propières, Quinié, Ranchal), à la sous-préfecture de Villefranche-sur-Saône, dans le Vignoble (trois rébellions à Belleville, une autre à Saint-Jean-d'Ardières) et dans le Val-de-Saône (Arnas). Autant de pays fort dissemblables, mais tous marqués, à des degrés divers, par le poids de la jeunesse. L'essor démographique est en effet fort en Montagne, il est encore soutenu dans le Vignoble, tandis que la croissance de Lyon et de ses faubourgs est alimentée par les flux migratoires. La solidarité entre jeunes existe partout ; seule varie la composition des groupes. En mars 1826, une quinzaine de compagnons tailleurs de pierres affrontent les gendarmes de Villefranche ; au faubourg Saint-Clair, en mars 1830, ce sont quinze garçons meuniers qui défient les gendarmes. ${ }^{(24)}$ Le plus souvent, il s'agit de jeunes paysans, dont les liens sont resserrés par la paroisse d'appartenance et parfois par la classe d'âge.

Ces jeunesses sont en effet fortement cloisonnées, d'où de fréquentes rixes intra et surtout intercommunales : le Rhône figure au nombre des départements qui se distinguent par la gravité des rixes. ${ }^{(25)}$ Celles-ci inquiètent d'autant plus les autorités qu'elles peuvent basculer dans la rébellion lors de l'intervention des gendarmes. L'arrestation d'un des adversaires constitue le casus belli le plus courant, mais la simple interposition de la brigade peut provoquer l'affrontement. Le 24 juin 1830, à la foire du hameau de Sanié, le brigadier et trois gendarmes de Beaujeu sont menacés aux cris de "Allez vous faire foutre, sacrées canailles, que venez-vous faire ici, vous allez voir tout à l'heure... ", lorsque, au début de l'aprèsmidi, ils tentent de séparer les jeunes gens de Marchampt et de Quinié, qui ont coupé des peupliers pour en faire des triques. ${ }^{(26)}$ En dépit de son caractère banal, le retournement de la violence contre les gendarmes reste paradoxal. Les jeunesses en conflit refondent, au moins momentanément, leur unité contre les gendarmes. Est-ce aussi simple ? Dans la majorité des cas, on constate que c'est seulement l'une des parties, celle qui parait avoir été en position de force ou d'agresseur qui lutte contre les gendarmes. Il s'agit alors de ne pas perdre le fruit d'une victoire acquise et de réaffirmer la maitrise du terrain sur les nouveaux venus.

L'autre modèle des rébellions juvéniles dans le Rhône est offert par les rébellions en résistance à une arrestation. Des jeunes peuvent prendre la tête d'un mouvement spontané comme à La Guillotière, le dimanche 10 septembre 1820, 
lorsqu'en pleine vogue un huissier assisté de la gendarmerie arrête un individu pour dettes au milieu de la foule. ${ }^{(27)}$ Le plus souvent, c'est un insoumis qu'il faut secourir. C'est le cas, par exemple, le 21 mai 1820 à Propières, où deux gendarmes de Monsols sont menacés par une vingtaine de jeunes gens qui empêchent l'arrestation d'un retardataire de la classe de 1817 de Saint-Igny-de-Vers.

\section{La conjonction des contentieux}

On ne saurait toutefois adopter une classification rigide. Un examen attentif des affaires dévoile la superposition des conflits qui concourent à l'affrontement ouvert. Ainsi en est-il de la rébellion qui éclate, deux jours après celle de Propières. Dans le hameau isolé de Montpinay (commune de Ranchal), se tient une foire annuelle spécialisée dans le louage des domestiques. C'est dire l'affluence de jeunes gens cherchant à s'embaucher, au nombre desquels figurent des insoumis qui ont trouvé refuge dans ce canton montagneux et boisé, frontalier du département de la Loire. La foire est d'ailleurs décriée pour les désordres qu'elle favoriserait (rixes et " débauche »). ${ }^{(28)}$ Ceux du 23 mai 1820 émeuvent cependant les autorités en raison des circonstances et de la violence de l'affrontement, dont témoigne le maréchal des logis de Thizy qui dirigeait trois brigades réunies pour l'occasion :

« $[P]$ lus de deux cent jeunes gens s'étaient réunis en cet endroit et s'y battaient, lors nous y sommes tout de suite transportés pour y mettre l'accord et rétablir l'ordre ; mais comme ce n'était qu'une ruse de la part des jeunes gens qui prétendaient nous attirer par ce moyen pour nous surprendre et se révolter contre nous, nous avons été à l'instant cernés par tous ces jeunes gens qui ont commencé à nous traiter de brigands et à nous lancer de grosses pierres en disant qu'ils allaient tous nous tuer, étant armés chacun d'un gros bâton carré, j'ai eu à leur dire que nous n'étions pas là pour nous battre avec eux, bien le contraire, pour les protéger et les empêcher qu'ils ne se fassent du mal entr'eux ; de faire attention que nous étions armés et disposés à repousser la force par la force, et que nous leur ordonnions, de la part du Roi, de se retirer sur le champ ; mais loin d'adhérer à mes paroles de paix et quoique je leur aie répété plusieurs fois force à la loi que nous allions repousser la force par la force, ils ont continué de nous lancer des pierres et à tomber sur nous comme des furieux. " (29)

(27) Bulletin de police des 22-24 septembre 1820, AN, F7 3793.

(28) « Ce point est le rendez-vous des domestiques et d'une grande partie de la jeunesse des deux sexes des communes environnantes sur un rayon de 3 à 4 lieues. La réunion y est immense, et il $\mathrm{y}$ règne depuis longtemps la licence la plus effrénée en tous genres. [...] le vrai but de la plupart de ceux qui s'y rendent est la débauche, le libertinage ou le désir de vider quelques querelles ou se faire une vengeance. On y danse, on y boit une partie du jour puis on se dissémine dans les bois taillis environnants : là des désordres de tous genres ont lieu de la manière la plus déshonnête et la plus scandaleuse. Sur le soir, quand les têtes sont bien échauffées par le vin, viennent les querelles et 
les batteries " : le juge de paix de Saint-Nizier-

d'Azergues au sous-préfet de Villefranche-sur-Saône, 4 juin 1820, A.D. Rhône, 4 M 229.

(29) Le maréchal des logis de Thizy au souspréfet de Villefranche-surSaône, 25 mai 1820, A.D. Rhône, 4 M 229.

(30) Le substitut du procureur général de Lyon au garde des Sceaux, 24 octobre $1820, \mathrm{AN}$, $\mathrm{BB}^{18} 1064$.
Aussi vivant soit-il, ce compte rendu n'est qu'une version des faits. La prise en considération d'autres points de vue permet de comprendre différemment cet événement. Il peut relever de la rixe intercommunale car, à l'origine, les gendarmes sont intervenus pour séparer ce qu'ils croyaient être un combat entre jeunesses rivales. La grande proportion des insoumis pousse cependant à y voir un épisode de la lutte contre le recrutement. D'autres causes se dessinent au fil des témoignages, comme la fronde antifiscale - des employés des impôts indirects sont agressés pendant l'assaut contre les gendarmes -, jusqu'à l'antagonisme entre gens du bourg et paysans, redoublé par la différence d'âge et peut-être la présence des femmes. Un incident significatif a en effet précédé l'échauffourée :

"Arrivé à Montpinay, le sieur Bellot [receveur ambulant des impôts indirects] se joignit au maréchal des logis de Thizy, au brigadier de Charlieu, au sieur Michaud médecin à Cours, au sieur Jacquet notaire et sa femme et à plusieurs femmes pour dîner sur l'herbe. Là ils furent provoqués par plusieurs jeunes paysans qui vinrent chanter autour d'eux des chansons insultantes. On crut apercevoir qu'ils en voulaient principalement à la Gendarmerie et au sieur Michaud. "(30)

Les formes mêmes de la rébellion sont sujettes à débat, car les témoins et les autorités l'interprètent soit comme un simple débordement lié à l'excès de boisson au cours d'une rixe spontanée, soit comme une simulation pour attirer les gendarmes dans un piège - ce qui renvoie à un complot local concerté par la jeunesse de Cours pour se venger de la brigade de Thizy après des tensions lors des précédentes opérations de recrutement -, soit enfin comme un épisode d'une conspiration politique d'ampleur nationale, comme le fait craindre la succession en quelques jours des atteintes à la force publique dans cet arrondissement.

La jeunesse des rebelles reste en définitive le seul dénominateur commun à toutes ces lectures. Une approche transversale de la vingtaine d'affaires rhodaniennes met au jour certaines circonstances qui, plus que des classements thématiques, en apprennent davantage sur la sensibilité des jeunes et les valeurs qu'ils ont à cœur de défendre. La solidarité du groupe, l'effet d'entraînement, mais aussi le vin et les filles, aboutissent à des désordres qui finissent par se retourner contre la brigade de service.

Par-delà l'anecdote, certains détails renvoient aux prérogatives communautaires dont les jeunes sont dépositaires. La réaction est brutale lorsque les 
gendarmes troublent les traditions, comme à la fête votive de Saint-Romainle-Popey, le 21 juillet 1816. Le lieutenant de gendarmerie de Villefranchesur-Saône arrache les plumets verts et rouges ornant le chapeau gansé en blanc de deux jeunes hommes parce qu'il y voit un rappel des trois couleurs. La riposte est violente : l'officier s'enfuit la tête ensanglantée ; un gendarme est roué de coups. Le ministère public relativise malgré tout l'événement en soulignant l'honneur doublement offensé des jeunes gens, anciens militaires, interpellés au milieu de la danse et humiliés en public. Le châtiment frappe par son mélange de vigueur et de paternalisme : cinq jeunes gens sont condamnés aux fers, mais leur grâce est conditionnée à la bonne conduite des habitants lors des prochaines fêtes. ${ }^{(31)}$

La répression vise d'abord en effet à prévenir le renouvellement de semblables désordres, ne serait-ce que localement, tant les effets des cycles vindicatifs peuvent être dangereux. Au fur et à mesure que se prolonge une querelle, elle dépasse les protagonistes initiaux. Cet élargissement est une donnée essentielle pour mieux saisir la nature des luttes entre jeunes gens et gendarmes : elle montre que la rébellion ouverte n'est souvent que la manifestation d'un conflit larvé et donne à voir les liens entre la jeunesse et la communauté locale.

\section{Jeunesses et communautés : la leçon des cycles vindicatifs}

Au dire du sous-préfet de Villefranche-sur-Saône, le canton du Bois-d'Oingt serait « un des plus difficiles à gouverner, la gendarmerie y est vue d'un assez mauvais œil ", notamment à cause du braconnage. ${ }^{(32)}$ C'est sur ce fond d'animosité qu'éclate, à partir de l'été 1820, une série d'incidents (agression des gendarmes conduisant un déserteur, résistance généralisée en faveur d'un individu ivre, etc.) entre la gendarmerie et la population de Chamelet, au sein de laquelle se distinguent les jeunes gens. Le climat se détériore au point qu'une grande enquête est lancée. Elle établit que ce conflit ne relève ni d'une gendarmophobie instinctive, ni d'un contentieux spécifique entre la brigade et la jeunesse. Il s'agit d'abord d'un conflit de pouvoir entre le maire et le brigadier, mais compliqué par les liens familiaux : le jeune homme le plus virulent est le capitaine des fêtes et le fils de l'adjoint. La jeunesse est instrumentalisée par le maire comme force vive, tout comme ces jeunes exploitent la rivalité entre les autorités

(31) Bureau des grâces. Dossiers de Bourquin, de Vial et des frères Renevier, Pierre, AN, $\mathrm{BB}^{21} 220^{1}$.

(32) Le sous-préfet de Villefranche-sur-Saône au préfet du Rhône, 29 août 1820, A.D. Rhône, 4 M 181.

(33) Sur cette affaire, dossier « Brigade de Chamelet. Altercation entre le maire et le brigadier ", A.D. Rhône, 4 M 181.

(34) Bureau des grâces. Dossier de Pigeot, Dumont et Fert (avril 1831), AN, BB 21358.

(35) Procès-verbal de l'adjoint de Belleville, 14 juin 1830, tribunal correctionnel de Villefranche-sur-Saône : dossier de procédure, A.D. Rhône, Uv 1205. 
(36) Procès-verbal des brigades de Villefranchesur-Saône et de La Croisée de Belleville, 16 juin 1830 , ibid.

(37) Jean-Claude Farcy, La jeunesse rurale dans la France du XIXème siècle, op. cit.

(38) Jean-Yves Carluer établit un premier bilan pour le Finistère, "Le gendarme, le maire et les Bas-Bretons. Quelques réflexions sur les représentations du pouvoir et la fragilité des épaulettes et des aiguillettes au XIXème ", Violence et société en Bretagne et dans les pays celtiques, colloque international, Brest, 18-

20 mars 1999, actes réunis par Jean-Yves Carluer, Brest, Kreitz 13, dans l'exercice de l'ordre public, pour se garantir plaisirs et libertés en obtenant une extension des horaires d'ouverture des cabarets. La jeunesse travaille ainsi à ses intérêts propres par-delà sa participation à la lutte de la communauté villageoise. Et si conflit de génération il y a, c'est davantage entre le maire, rompu aux affaires municipales depuis quinze ans, et le brigadier, récemment admis à ce grade et dans la gendarmerie, qui entend se faire respecter. Ce jeune chef de brigade n'est d'ailleurs pas coupé de la jeunesse, puisque celle du Chamelet lui reproche ses parties de chasse avec les jeunes des environs. ${ }^{(33)}$

L'affaire suivante est aussi simple que significative. Le 10 juin 1830, à Belleville, chef-lieu de canton de 2.400 habitants de l'arrondissement de Villefranche-sur-Saône, le brigadier de La Croisée interpelle un jeune homme qui, se destinant par goût à l'état militaire, se laissait pousser la moustache. «Voilà donc cette moustache naissante, lui dit le brigadier ; dans un an, répond le jeune homme, elle saura se faire respecter. " À cette réponse, le brigadier, furieux, appelle ses subordonnés. Il s'ensuit une rixe violente au cours de laquelle les gendarmes tirent leurs sabres et blessent un jeune homme. ${ }^{(34)}$ Trois jours plus tard, les mêmes jeunes gens exécutent leur vengeance. Cette fois, c'est Pierre Fert, le remplaçant outragé, voiturier de 24 ans, qui insulte dans la soirée le brigadier François Samogneux, âgé de 61 ans, avant de le frapper. Le sous-officier, assisté de deux gendarmes, l'arrête ; en réaction, la jeunesse en masse exige sa libération et menace la brigade. Les efforts conjugués de l'adjoint, du capitaine des pompiers et de quelques particuliers évitent l'affrontement, mais un conseiller municipal doit raccompagner à leur résidence les gendarmes pour prévenir le risque d'embuscade sur le chemin du retour. ${ }^{(35)}$

L'affaire cesse d'être le propre des jeunes gens. Ceux-ci ont su rallier à leur cause de nombreux habitants choqués par les brutalités des gendarmes. Lorsque Fert est arrêté chez lui, le 16 juin, par les brigades de Villefranche et de La Croisée, il prend de haut les gendarmes, leur déclarant que ses camarades et lui " avaient des grosses têtes pour eux qui les soutiendraient ». ${ }^{(36)}$ Des bruits de vengeance se répandent ; un ancien gendarme, retiré à La Croisée, est même menacé. La révolution de Juillet met un terme au conflit avec la remise de peines des trois principaux condamnés et le départ en retraite du brigadier. 
Que conclure de ces rébellions juvéniles ? Leur nombre suffit à attirer l'attention sur leur place spécifique au sein du répertoire rébellionnaire d'ensemble. L'âge des individus aux prises avec la gendarmerie apporte un précieux éclairage : sans conditionner l'affrontement, il en colore les formes. À bien des égards, ces rébellions juvéniles participent au conflit global, mais circonscrit à une minorité de départements, qui oppose les villages et l'État dans la première moitié du siècle. Problème complexe au demeurant, car les jeunes sont autant un ferment de dissolution individualiste de la communauté que son ardent défenseur, tandis que l'État apparaît aussi bien comme l'adversaire de la jeunesse dont il entend contrôler, voire effacer, les manifestations traditionnelles (comme le charivari), tout en conférant à cette jeunesse, fragmentée il est vrai en classes d'âge, une identité forte avec les conscrits. ${ }^{(37)}$

Les questionnements que suscitent les rébellions juvéniles dépassent d'ailleurs cette problématique. On ne saurait nier le caractère ostentatoire de ces affrontements physiques dans le cadre de circonstances festives. À la lumière des récits présentés, il convient de souligner le poids des rivalités viriles. Comment en effet ne pas remarquer l'influence du regard des femmes, à Blanzat comme à Montpinay, ou encore la volonté de rabaisser l'adversaire à travers des symboles frappants, qu'il s'agisse de plumets arrachés, de moustache moquée ou des insignes de l'autorité arrachés aux gendarmes ? ${ }^{(38)}$ En luttant contre la brigade, les jeunes gens ne défendent pas seulement l'ordre villageois : ils y conquièrent leur place comme au terme d'un rituel extrême, affirmant leur force d'homme face aux gendarmes. Ces derniers n'incarnent-ils pas un modèle de masculinité affirmée (la haute stature, l'uniforme, le port d'arme ou le cheval) ? (39) L'effacement des rébellions dans la seconde moitié du XIXème siècle, à la faveur de la décrispation des relations entre les villages et l'État, est également lié, en ce qui concerne la jeunesse, à un relâchement de la pression démographique, mais aussi à la réorientation des valeurs de la gendarmerie et l'abandon d'un modèle guerrier et parfois provocateur, ${ }^{(40)}$ alors même que la généralisation progressive du service militaire contribue à faire retomber le besoin d'affirmation conflictuelle de la jeunesse.
2000, p. $505-521$.

(39) Voir Gil Mihaely,

L'émergence du modèle militaro-viril au XIXème siècle, doctorat, EHESS, sous la direction de Christophe Prochasson, 2004, 455 p.

(40) Le doctorat d'Arnaud-Dominique Houte permet de suivre, en relais du nôtre, les mutations de la figure du gendarme et de son insertion sociale : Le métier de gendarme national de la Monarchie de Juillet à la Grande Guerre. La construction d'une identité professionnelle (I840-I9I4), sous la direction de Jean-Noël Luc, Paris IV- 